\title{
Scalable Analysis Techniques for Microprocessor Performance Counter Metrics
}

D.H. Ahn, J.S. Vetter

This article was submitted to

SC2002: High Performance Networking and Computing, Baltimore, Maryland, November 16-22, 2002

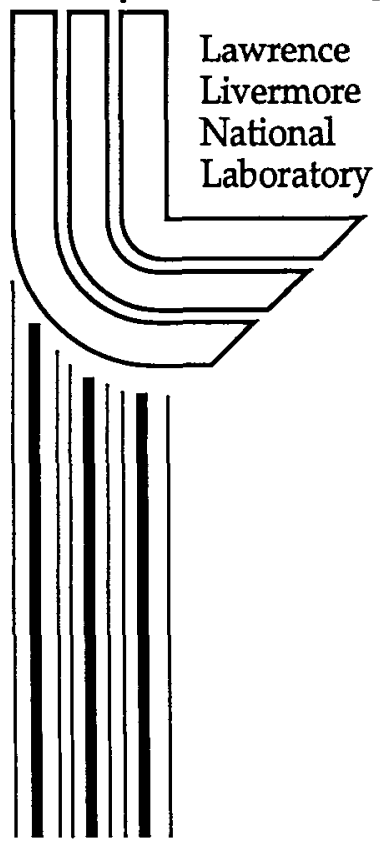

July 24, 2002 


\section{DISCLAIMER}

This document was prepared as an account of work sponsored by an agency of the United States Government. Neither the United States Government nor the University of California nor any of their employees, makes any warranty, express or implied, or assumes any legal liability or responsibility for the accuracy, completeness, or usefulness of any information, apparatus, product, or process disclosed, or represents that its use would not infringe privately owned rights. Reference herein to any specific commercial product, process, or service by trade name, trademark, manufacturer, or otherwise, does not necessarily constitute or imply its endorsement, recommendation, or favoring by the United States Government or the University of California. The views and opinions of authors expressed herein do not necessarily state or reflect those of the United States Government or the University of California, and shall not be used for advertising or product endorsement purposes.

This is a preprint of a paper intended for publication in a journal or proceedings. Since changes may be made before publication, this preprint is made available with the understanding that it will not be cited or reproduced without the permission of the author.

This report has been reproduced directly from the best available copy.

Available electronically at http://www.doe.gov/bridge

Available for a processing fee to U.S. Department of Energy and its contractors in paper from

U.S. Department of Energy

Office of Scientific and Technical Information

P.O. Box 62

Oak Ridge, TN 37831-0062

Telephone: (865) 576-8401

Facsimile: (865) 576-5728

E-mail: reports@adonis.osti.gov

Available for the sale to the public from

U.S. Department of Commerce

National Technical Information Service

5285 Port Royal Road

Springfield, VA 22161

Telephone: (800) 553-6847

Facsimile: (703) 605-6900

E-mail: orders@ntis.fedworld.gov

Online ordering: http://www.ntis.gov/ordering.htm

OR

Lawrence Livermore National Laboratory

Technical Information Department's Digital Library

http://www.llnl.gov/tid/Library.html 


\title{
Scalable Analysis Techniques for Microprocessor Performance Counter Metrics
}

\author{
Dong H. Ahn \\ Jeffrey S. Vetter \\ Lawrence Livermore National Laboratory \\ Livermore, CA, USA 94551 \\ $\{$ vetter3, ahnl\}@IlnI.gov
}

\section{Introduction}

Contemporary microprocessors provide a rich set of integrated performance counters that allow application developers and system architects alike the opportunity to gather important information about workload behaviors. These counters can capture instruction, memory, and operating system behaviors. Current techniques for analyzing data produced from these counters use raw counts, ratios, and visualization techniques to help users make decisions about their application source code.

While these techniques are appropriate for analyzing data from one process, they do not scale easily to new levels demanded by contemporary computing systems. Indeed, the amount of data generated by these experiments is on the order of tens of thousands of data points. Furthermore, if users execute multiple experiments, then we add yet another dimension to this already knotty picture. This flood of multidimensional data can swamp efforts to harvest important ideas from these valuable counters.

Very simply, this paper addresses these concerns by evaluating several multivariate statistical techniques on these datasets. We find that several techniques, such as statistical clustering, can automatically extract important features from this data. These derived results can, in turn, be feed directly back to an application developer, or used as input to a more comprehensive performance analysis environment, such as a visualization or an expert system.

\section{Microprocessor Hardware Performance Counters}

Modern microprocessors include integrated hardware support for non-intrusive monitoring of a variety of processors and memory system events, commonly referred to as hardware counters [3,9]; this capability is very useful to both computer architects [2] and applications developers [15]. These counters fill a gap that lies between detailed microprocessor simulation and software instrumentation. Software instrumentation can introduce perturbation into an application and the measurement process itself. On the other hand, these counters have relatively low perturbation and can provide insightful information about processor and memory-system behavior. Even though this information is statistical in nature, it does provide a window into certain behaviors that are realistically impossible to gather otherwise. For instance, on IBM's Power3 microprocessor, these events include various categories of instructions, cache misses, branch predictions, memory coherence operations, and functional unit utilization.

Several tools and microprocessors have added additional functionality to simple event counting. Intel's Itanium processors [6] have features that allow monitoring based 
on an instruction address range, a specific instruction opcode, a data address range, and/or the privilege level. In addition, the Itanium supplies event address registers that record the instruction and data addresses of data cache misses for loads, the instruction and data addresses of data TLB misses, and the instruction addresses of instruction TLB and cache misses.

DEC/Compaq implemented another useful strategy for hardware counters: instruction sampling within the microprocessor. Using this approach, a performance-monitoring tool, such as ProfileMe [4] or DCPI [1], could arbitrarily choose to measure performance characteristics as they flowed through the processor pipeline. The tool could, then, gather this information over the execution of an application and attribute performance problems to certain instructions.

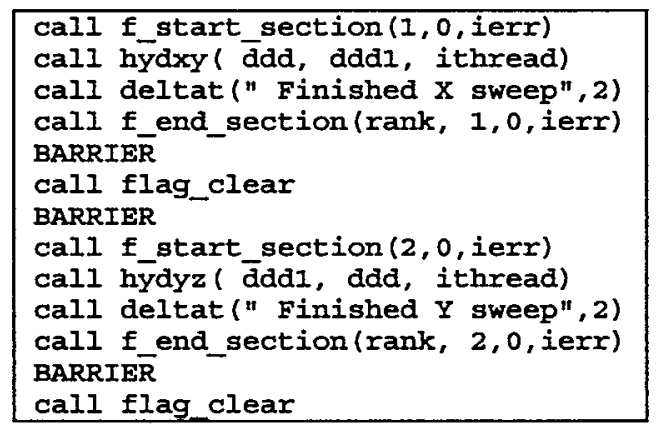

Figure 1: Sample code segment from function runhyd3 of sPPM.

\subsection{Counting Hardware Events}

Our approach to using hardware counters rests on bracketing targeted code regions with directives that program the counters to capture events of interest, start and stop the counters, and store the counter values. Users can insert these directives several ways: manually or by using a compiler, a binary editor, or dynamic instrumentation. Hardware counters do require the appropriate operating system and library support to attribute counts appropriately to the proper processes and threads.

\begin{tabular}{|c|c|c|c|c|c|c|c|c|c|c|}
\hline$\supseteq$ & & & \multicolumn{8}{|c|}{ Counter value } \\
\hline 0 & 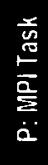 & 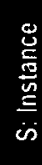 & 1 & 2 & 3 & 4 & 5 & 6 & 7 & 8 \\
\hline 1 & 1 & 1 & 8305760504 & 795651387 & 2265349817 & 14689488 & 72993923 & 3744267304 & 2123235784 & 1253921843 \\
\hline 1 & 1 & 2 & 8233114700 & 7587713598 & 2257442295 & 8987587 & 72816919 & 3612932116 & 2100752913 & 1260163691 \\
\hline 1 & 2 & 1 & 8197360363 & 7701750765 & 2233070347 & 14695959 & 73425197 & 3736956914 & 2075722824 & 1237231534 \\
\hline 1 & 2 & 2 & 8135138668 & 7593760051 & 2207456335 & 9172755 & 73699055 & 3590374684 & 2060311042 & 1230463969 \\
\hline 2 & 1 & 1 & 8326329304 & 7559198564 & 2401195595 & 14583869 & 72382972 & 3717326869 & 2078653081 & 1233604083 \\
\hline 2 & 1 & 2 & 8291791110 & 7421248463 & 2334628952 & 8509892 & 72074918 & 3540521698 & 2060023498 & 1230670879 \\
\hline 2 & 2 & 1 & 8405106757 & 7645055689 & 2415396992 & 14655896 & 72785214 & 3708798229 & 2104739801 & 1248538508 \\
\hline 2 & 2 & 2 & 8381061956 & 7523753702 & 2377276028 & 8606055 & 72608329 & 3553288776 & 2084495857 & 1256915516 \\
\hline
\end{tabular}

Table 1: Counter values from code segment.

Figure 1 shows a code segment from SPPM [11] that has been instrumented with high level library routines written on top of MPX [10] and PAPI [3] in order to capture eight hardware counter values: total processor cycles, total instructions, cycles stalled waiting 
for memory accesses, floating point divide instructions, L1 cache misses, floating point instructions, load instructions, and store instructions.

As Figure 1 illustrates, every execution of this sequential code segment will generate one instance of counter values for each MPI task. Therefore, applications that execute this code segment millions of times will generate millions of instances of counter values. Table 1 shows the raw counter value table that is generated from the code segment in Figure 1, using two MPI tasks. The $G$ column lists the instrumentation identifiers that represent different regions of the code. The $S$ column lists instances of these regions. Clearly, in real experiments, this data management problem can become intractable!

\section{Multivariate Statistical Techniques for Performance Data}

As we illustrated in Section 2, each instrumentation point for an application can generate a vast number of hardware counter values. Multiple experiments can aggravate this issue even further. To analyze this data, we turn to multivariate statistical techniques to help focus the user's attention on the important metrics and the distribution of those metrics.

\subsection{Performance Metric Spaces}

For further analysis, we model these values as points in a multidimensional space. To make this notion more formal, consider a set of $k$ dynamic performance metrics, hardware counters in our case, measured on a set of $P$ parallel tasks, on a set of $g$ instrumentation regions, and on $s$ samples. Abstractly, one can then view these events as defining a collection of these points that describe parallel system characteristics. Following [14], if $R_{i}$ denotes the range of metric $k$, we call the Cartesian product

$$
M=R_{1} \times R_{2} \times \ldots \times R_{\mathrm{k}}
$$

a performance metric space. Thus, the ordered $k$-tuples

$$
\left(v_{1} \in R_{1} ; v_{2} \in R_{2} ; \ldots ; v_{\mathrm{k}} \in R_{\mathrm{k}}\right)
$$

are points in $M$. It is important to note that this definition of the metric space does not include the dimensions of instrumentation identifier, parallel task identifier, or measurement instance. Furthermore, this model assumes that this higher-dimension data can be down-sampled into this space as appropriate. For instance, we collect all the points for one instrumentation region across all tasks and across all measurements and then project it into this metric space. This situation would generate $k \times P \times s$ points. While this trivial example illustrates our formalization, we expect to use our techniques on much larger systems where $k>10, g>10, P>>10$, and $s \gg 10$.

The goal of our analysis techniques is now clear; we must reduce this massive number of measurement points and the dimensionality of the metric space to a comprehendible scale. Traditional multivariate statistical techniques warrant investigation as vehicles for understanding this data. In fact, projection pursuit [14] and clustering [12] have been applied to understanding real-time performance data; this previous work strongly suggests that such techniques will be useful for managing hardware counter data. These multivariate statistical techniques allow users to draw inferences from observations with multiple variables (dimensions) and they include dimension reduction and classification. 


\subsection{Data Preparation}

Raw data as generated by reading the hardware counters directly can provide useful information; however, in the context of performance analysis, derived metrics are important. For example, the raw metric for number of cycles supplies a useful estimate of how long a code region executed; however, the derived metric of number of instructions divided by the number of cycles (IPC or instructions per cycle) can directly emphasize code regions that are performing poorly. On the other hand, raw metrics are necessary to help gauge the overall importance of code regions per se. For instance, the IPC of a code region that accounts for only minuscule numbers of cycles during the application execution is irrelevant.

\subsection{Clustering}

Clustering is a rudimentary, exploratory technique that is helpful in understanding the complex nature of multivariate relationships [7]. It provides a familiar means for assessing dimensionality, detecting outliers, and suggesting attractive hypotheses about relationships between the data. Cluster analysis makes no assumptions about the number of clusters or the cluster structure. It relies only on a metric that calculates the similarities or distances between data points. There have been a wide variety of clustering algorithms proposed. Major differences are whether particular methods simply partition data points into a given number of groups or build more complicated cluster (or data point) hierarchies.

In the context of hardware counter data, we propose both hierarchical and nonhierarchical methods will help users identify equivalence classes of data points and an 'important' subset of entire performance metrics that make more contribution to the existence of those classes.

We demonstrate how hierarchical algorithms give users insights about overall cluster structure of a data set by means of dendrogram, while nonhierarchical methods, such as the $\mathbf{k}$-means algorithm, provide an efficient method to explain the importance of each metric on a cluster configuration by using F-ratio of each metric (Section 4.4). F-ratio is a technique for univariate analysis of variance that is defined as $\frac{\text { Between-ClusterVariablity }}{\text { Within-ClusterVariablity }}$.

Hence, metrics that vary greatly among different clusters and remain the same in the same cluster yields higher F-ratio.

$\mathrm{K}$-means and F-ratio can also be employed when the decision on number of clusters is not obvious. This situation happens often when users do not have reasonable prior knowledge about target application's behavior. K-mean and F-ratio methods provide a means by which a system can automatically partition data points into a number of clusters as to maximize the between-cluster variability relative to the within-cluster variability.

\subsection{Factor Analysis}

Factor analysis is a multivariate technique that makes it possible to describe the covariance relationships among many variables in terms of a few underlying quantity, factors. In the context of hardware counter space, we propose it will reduce the dimensionality of our performance metric space, $\mathrm{M}=\left[R_{1} \times R_{2} \times \ldots \times R_{\mathrm{k}}\right]$, by assembling highly correlated metrics in a peer group while separating uncorrelated ones into the other groups. (e.g. $\left[R_{a}, R_{b}, R_{c}\right], \mathrm{x}\left[R_{d}\right] \mathrm{x} \ldots \mathrm{x}\left[R_{i}, R_{j} R_{k}\right]$ ). This grouping can guide users to choose a right set of metrics for refining their code optimization efforts. 
In the factor analysis model, our metrics space $M$ can be rewritten as

$$
\begin{aligned}
& R_{1}-v_{1}=l_{11} F_{1}+l_{12} F_{2}+\ldots+l_{1 m} F m+\varepsilon_{1} \\
& R_{2}-v_{2}=l_{21} F_{1}+l_{22} F_{2}+\ldots+l_{2 m} F m+\varepsilon_{2} \\
& \vdots \\
& R_{p}-v_{p}=l_{p 1} F_{1}+l_{p 2} F_{2}+\ldots+l_{p m} F m+\varepsilon_{p}
\end{aligned}
$$

Where $F_{i}$ is $i^{\text {th }}$ common factor, $R_{j} j^{\text {th }}$ metrics, $v_{k}$ mean of $R_{j}$, and coefficient $l_{j i}$ is the loading of $R_{j}$ on the factor $F_{i}$. As this notation suggests, grouping $\mathrm{R}$ 's that have higher loadings for a particular $F$ will yield a group whose $R$ 's are highly correlated.

\subsection{Principal Component Analysis}

define.

\section{Evaluation}

We empirically evaluated our techniques on an operational prototype with two applications. We scale each application up to 128 tasks. As Figure 1 illustrates, we first instrument the application and collect hardware counter data on the target platform. We then clean, merge, and prepare this data for statistical analysis. Next, we apply several statistical techniques to the prepared data. In the future, we expect to feed the results from these analyses into a comprehensive performance analysis environment or automated performance tool.

\subsection{Instrumentation and Data Collection}

We manually instrument our target applications with source code annotations. Each instrumentation point identifies a code region to capture hardware counter metrics as Figure 1 illustrates. Hence, each application has $g$ instrumented code regions as defined in Section 3.1. For these experiments, we assume that each region captures the same set of $k$ hardware metrics.

In this framework, our tool can either write the each sample for each region to a tracefile during execution or accumulate the samples for each region and write the accumulated metrics to a file at termination. In the former context, tracefiles would grow at a rate proportional to $k \times g \times s$ for each parallel task. We implemented both modes, but still, we use the latter technique, which generates only $k \times g$ measurement points for each parallel task, here to prevent an explosion of data and measurement overhead in the application. Our statistical techniques remain valid for accumulated data; however, this selection has the drawback that accumulated measurements can hide certain performance phenomena.

At termination of the application experiment, each parallel task $P$ generates a local file. Our prototype merges these $P$ local files into one global file, containing all accumulated measurements for an application, and having size proportional to $k \times g \times P$. With all these raw metrics for one application now in one file, we can easily apply our statistical techniques to this file with a filter. This filter also manipulates the raw metrics for data cleaning and generating useful derived metrics as described in Section 3.2. 


\subsection{Platform}

We ran our tests on an IBM SP system, located at Lawrence Livermore National Laboratory. This machine is composed of sixteen $222 \mathrm{MHz}$ IBM Power3 8-way SMP nodes, totaling 128 CPUs. Each processor has three integer units, two floating-point units, and two load/store units. Its $64 \mathrm{~KB} \mathrm{L1}$ cache is 128 way associative with 32 byte cache lines and L1 uses a round-robin replacement scheme. The L2 cache is $8 \mathrm{MB}$ in size, which is four-way set associative with its own private cache bus. At the time of our tests, the batch partition had 15 nodes and the operating system was AIX 4.3.3. Each SMP node contains 4GB main memory for a total of 64 GB system memory. A Colony switch--a proprietary IBM interconnect--connects the nodes. We compiled the various tests with the IBM XI and KAI Guide compilers using IBM's MPI library in user-space mode. Our test jobs ran on dedicated nodes, although other jobs were concurrently using the network.

\subsection{Applications}

We evaluate our proposed techniques on two scalable applications. Each application has different computational and communication characteristics [13]. SPPM, for example, has large blocks of floating point computation with infrequent, large messages, while SMG2000 is at the other end of the spectrum, having frequent, small messages with smaller blocks of computation.

SPPM [11] solves a 3-D gas dynamics problem on a uniform Cartesian mesh, using a simplified version of the Piecewise Parabolic Method. The algorithm makes use of a split scheme of $\mathrm{X}, \mathrm{Y}$, and $\mathrm{Z}$ Lagrangian and remap steps, which are computed as three separate sweeps through the mesh per timestep. Message passing provides updates to ghost cells from neighboring domains three times per timestep. OpenMP provides threadlevel parallelism within MPI tasks.

Sweep3D $[5,8]$ is a solver for the 3-D, time-independent, particle transport equation on an orthogonal mesh and it uses a multidimensional wavefront algorithm for "discrete ordinates" deterministic particle transport simulation. Sweep3D benefits from multiple wavefronts in multiple dimensions, which are partitioned and pipelined on a distributed memory system. The three dimensional space is decomposed onto a two-dimensional orthogonal mesh, where each processor is assigned one columnar domain. Sweep3D exchanges messages between processors as wavefronts propagate diagonally across this 3-D space in eight directions. 


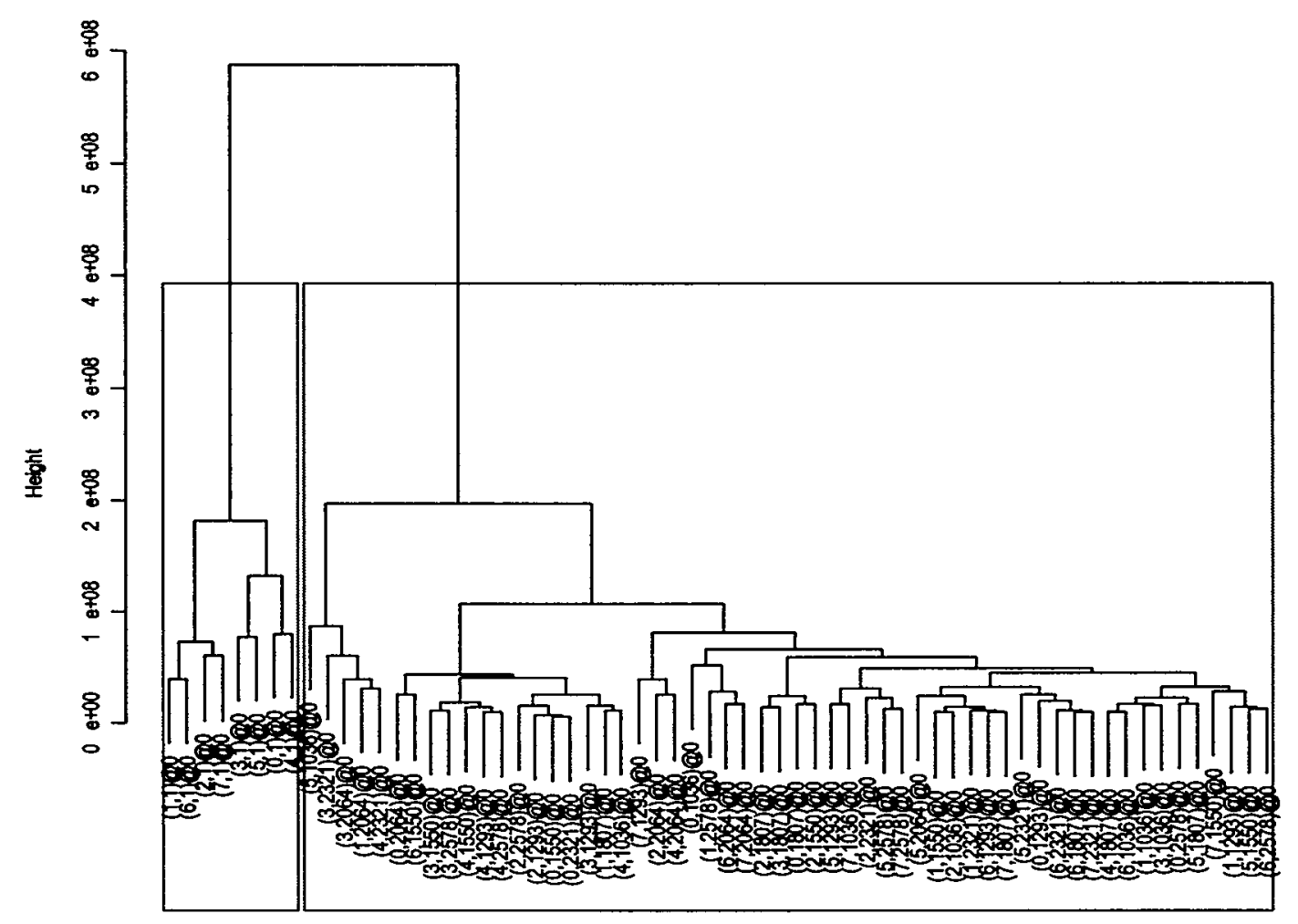

Figure 2: Dendrogram for a section of SPPM using raw metrics.

\subsection{Clustering}

\subsubsection{Agglomerative Hierarchical Method}

This method gives users insights about overall cluster structure that exist in a data space by constructing dendrograms. Figure 2 shows the dendrogram for one instrumented section of an SPPM experiment with 8 MPI tasks and 8 OpenMP threads per task. Since sPPM exploits parallelism with message passing for inter-node communication and OpenMP within shared memory for thread level parallelism, it is expected to have at least two natural clusters. AHM clearly identifies in Figure 2 the existence of two classes; one housing all 56 slave threads and the other cluster containing the 8 master threads.

Figure 3 illustrates the dendrogram of the same section of sPPM using derived metrics. As expected, the configuration does not change much from Figure 2, suggesting that the two clusters are performing similarly and any changes to code for either the master thread or the slave thread will propagate to its peers. Statistical techniques only with raw metrics would not immediately provide this type of perspectives.

Figure 4 shows the dendrogram for the raw metrics of a section of Sweep3d for an experiment using 64 MPI tasks. In this case, three clusters are sufficiently different. Our initial results show that because Sweep3d decomposes the global 3-D problem onto a 2-D orthogonal mesh for processor assignment, the assignment creates two different equivalence classes: one for internal processors (right), and one for corner and edge processors (center and left). Other techniques, such as projecting clusters (and data points) into principal component space, should be used to identify cluster configuration. 


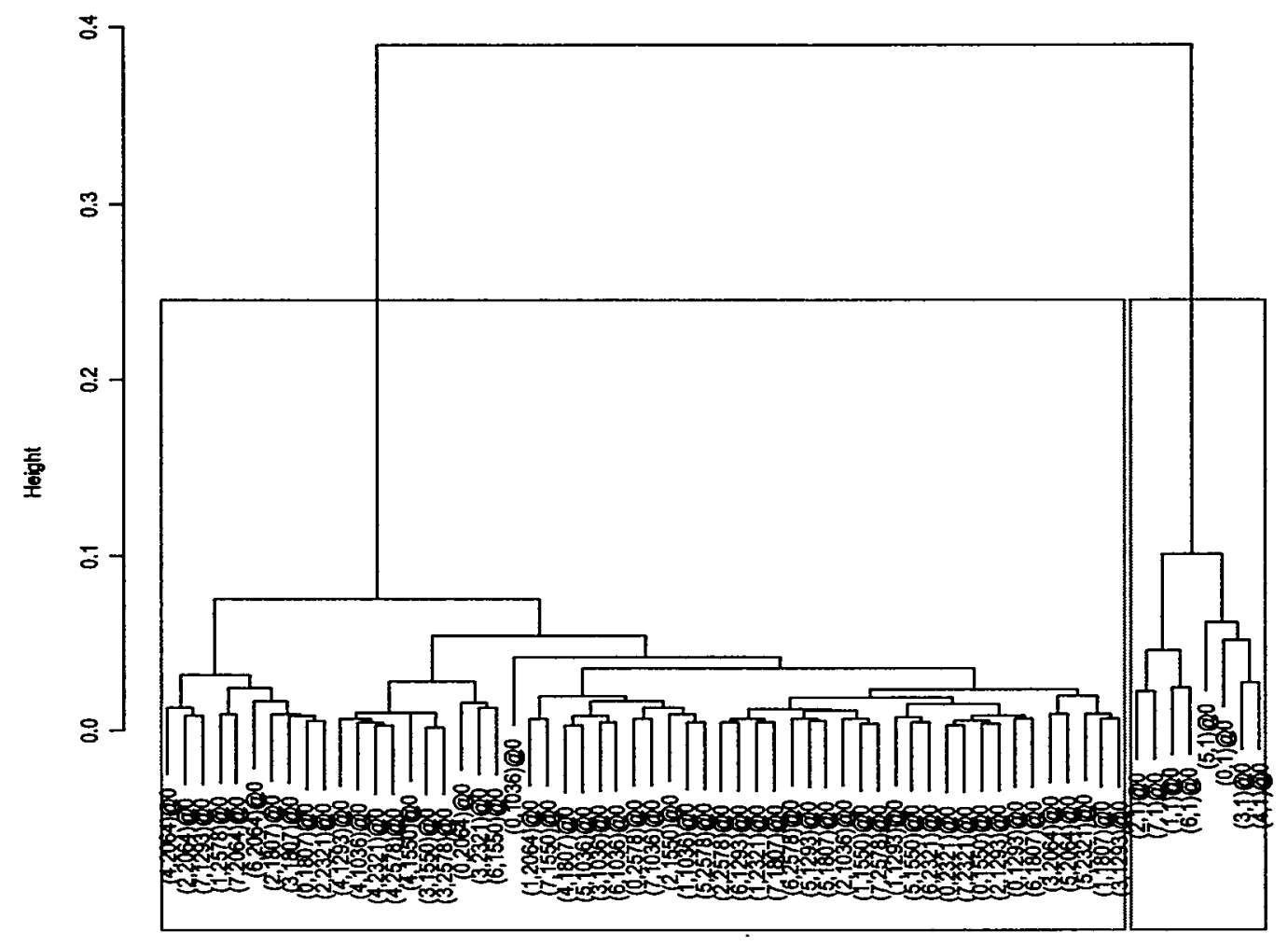

Figure 3: Dendrogram for a section of sPPM using derived metrics

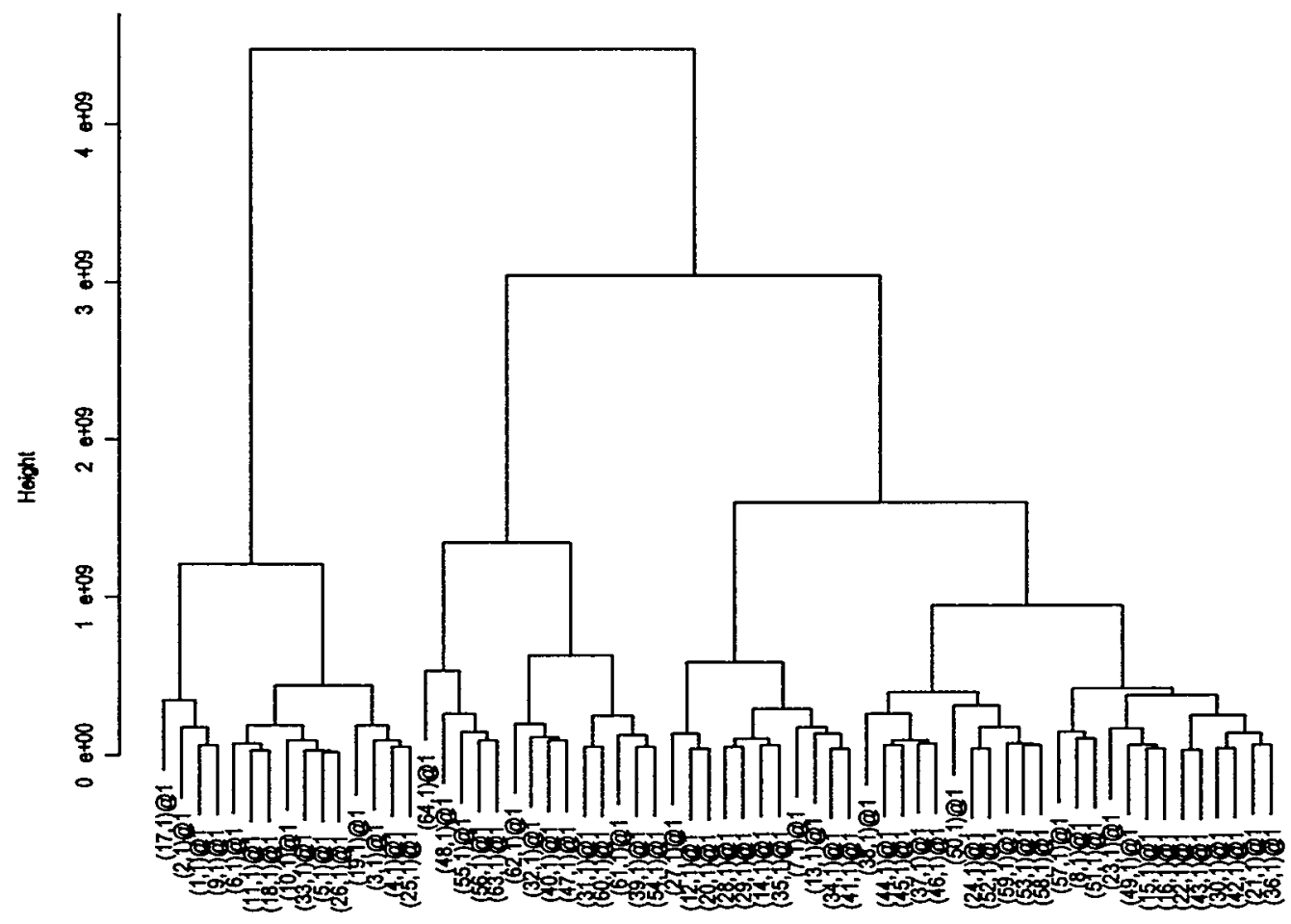

Figure 4: Dendrogram for sweep section of sweep3d using raw metrics. 


\subsection{2 k-means clustering and F-ratio}

While AHM gives a general idea about cluster structure, it is not entirely convenient to compare clusters and compute the importance of an individual metrics that yield the particular cluster configuration. Using k-means clustering and F-ratios, we ordered metrics for the same section on sPPM by their F-ratio in Table 2. It suggests that PAPI_MEM_SCY (Cycles Stalled Waiting for Memory Access), PAPI_SR_INS (Store instructions executed), and PAPI_L1_TCM (L1 total cache misses), are the three major reasons that we have two distinct clusters.

\begin{tabular}{lr}
\multicolumn{1}{c}{ Metrics } & F-ratio \\
PAPI.MEM.SCY & $\mathbf{7 . 9 1 4 2 5 5}$ \\
PAPI.SR.INS & $\mathbf{5 . 1 2 4 0 5 0}$ \\
PAPI.L1.TCM & $\mathbf{5 . 0 9 7 6 9 3}$ \\
PAPI.TOT.IIS & 3.488066 \\
PAPI.LD.INS & 3.473341 \\
PAPI.FP.INS & 2.498332 \\
PAPI.TOT.CYC & 1.673567 \\
PAPI.FDV.INS & 0.161107
\end{tabular}

Table 2: Metrics ordered by F-ratio size for a section of sPPM.

\subsection{Factor Analysis}

Table 3 shows the result of factor analysis on the same section of sPPM. Each column represents loadings of metrics for each factor. As it suggests, it is reasonable to group together those metrics with bigger loadings per column.

$\begin{array}{llll}\text { Metric } & \text { Factor1 } & \text { Factor2 Factor3 } \\ \text { PAPI.LD.INS } & \mathbf{- 0 . 6 5 8} & \mathbf{0 . 5 1 9} & \mathbf{0 . 5 4 1} \\ \text { PAPI.BR.PRC } & 0.000 & 0.000 & \mathbf{0 . 7 9 2} \\ \text { PAPI.L1.DCM } & \mathbf{0 . 9 6 9} & 0.13 & -\mathbf{0 . 1 6 1} \\ \text { PAPI.FXU.IDL } & -0.244 & \mathbf{0 . 8 8 3} & -0.290 \\ \text { PAPI.BTAC.M } & \mathbf{0 . 7 9 9} & 0.000 & 0.324 \\ \text { PAPI.FP.INS } & \mathbf{- 0 . 9 7 4} & 0.199 & 0.000 \\ \text { PAPI.MEM.WCY } \mathbf{0 . 9 5 7} & 0.000 & 0.000 \\ \text { PAPI.SR.INS } & 0.000 & \mathbf{0 . 6 9 0} & 0.000\end{array}$

Table 3: Factor Analysis of SPPM (with three factors assumed).

By setting a somewhat arbitrary threshold, 0.5, we are able to make four groups: \{PAPI.L1.DCM, PAPI.BTAC.M, PAPI.FP.INS, PAPI.MEM.WCY\}, \{PAPI.FXU.IDL, PAPI.SR.INS\}, \{PAPI.BR.PRC $\}$ and PAPI.LD.INS. We verified the metrics in the same group are highly correlated by scanning correlation matrix. Users can now select a representative metric per group for the further analysis.

\section{Conclusions}

Scalable computing platforms will generate tremendous volumes of performance data, especially when monitoring low-level, frequent events like those produced by microprocessor performance counters. Developers will need new techniques to help them gain insight into this massive dataset. Traditional multivariate statistical techniques can 
play a prominent role in this effort by reducing the dataset dimensionality and classifying similar datapoints. Our experiments on several applications demonstrate the feasibility of this approach and highlight several useful implementation strategies. For example, our experiments with sPPM and Sweep3d clearly confirmed that clustering on both raw and derived metrics can allow a user to understand the performance implications across all tasks in the application. We hope to feed results from this collection of statistical analysis techniques directly to the user, or to a sophisticated performance analysis system, such as a visualization or an expert system.

\section{Acknowledgments}

This work was performed under the auspices of the U.S. Department of Energy by the University of California, Lawrence Livermore National Laboratory under contract No. W-7405-Eng-48. This paper is available as LLNL Technical Report UCRL-JC148058.

\section{Appendix A: PAPI Power3 Hardware Events}

\begin{tabular}{|c|c|}
\hline ame & \\
\hline PAPI_L1_DCM & Level 1 data cache misses (PM_LD_MISS_L1,PM_ST_L1MISS) \\
\hline PAPI_L1_ICM & Level 1 instruction cache misses (PM_IC_MISS) \\
\hline PAPI_L1_TCM & Level 1 cache misses (PM_IC_MISS,PM_LD_MISS_L1,PM_ST_L1MISS) \\
\hline PAPI_CA_SNP & Requests for a snoop (PM_SNOOP) \\
\hline PAPI_CA_SHR & Requests for exclusive access to shared cache line (PM_SNOOP_E_TO_S) \\
\hline PAPI_CA_ITV & Requests for cache line intervention (PM_SNOOP_PUSH_INT) \\
\hline PAPI_BRU_IDL & Cycles branch units are idle (PM_BRU_IDLE) \\
\hline PAPI_FXU_IDL & Cycles integer units are idle (PM_FXU_IDLE) \\
\hline PAPI_FPU_IDL & Cycles floating point units are idle (PM_FPU_IDLE) \\
\hline PAPI_LSU_IDL & Cycles load/store units are idle (PM_LSU_IDLE) \\
\hline PAPI_TLB_TL & Total translation lookaside buffer misses (PM_TLB_MISS) \\
\hline PAPI_L1_LDM & Level 1 load misses (PM_LD_MISS_L1) \\
\hline PAPI_L1_STM & Level 1 store misses (PM_ST_L1MISS) \\
\hline PAPI_L2_LDM & Level 2 load misses (PM_LD_MISS_EXCEED_L2) \\
\hline PAPI_L2_STM & Level 2 store misses (PM_ST_MISS_EXCEED_L2) \\
\hline PAPI_BTAC_M & Branch target address cache misses (PM_BTAC_MISS) \\
\hline PAPI_PRF_DM & Data prefetch cache misses (PM_PREF_MATCH_DEM_MISS) \\
\hline PAPI_TLB_SD & Translation lookaside buffer shootdowns (PM_TLBSYNC_RERUN) \\
\hline PAPI_CSR_FAL & Failed store conditional instructions (PM_RESRV_CMPL) \\
\hline PAPI_CSR_SUC & Successful store conditional instructions (PM_ST_COND_FAIL) \\
\hline PAPI_CSR_TOT & Total store conditional instructions (PM_RESRV_RQ) \\
\hline PAPI_MEM_SCY & $\begin{array}{l}\text { Cycles Stalled Waiting for memory accesses } \\
\text { (PM_CMPLU_WT_LD,PM_CMPLU WTSST) }\end{array}$ \\
\hline PAPI_MEM_RCY & Cycles Stalled Waiting for memory Reads (PM_CMPLU_WT_LD) \\
\hline PAPI_MEM_WCY & Cycles Stalled Waiting for memory writes (PM_CMPLU_WT_ST) \\
\hline PAPI_STL_ICY & Cycles with no instruction issue (PM_OINST_DISP) \\
\hline PAPI_STL_CCY & Cycles with no instructions completed (PM_OINST_CMPL) \\
\hline PAPI_BR_CN & Conditional branch instructions (PM_CBR_DISP) \\
\hline PAPI_BR_MSP & Conditional branch instructions mispredicted (PM_MPRED_BR_CAUSED_GC) \\
\hline PAPI_BR_PRC & Conditional branch instructions correctly predicted (PM_BR_PRED) \\
\hline
\end{tabular}




\begin{tabular}{|l|l|}
\hline PAPI_FMA_INS & FMA instructions completed (PM_EXEC_FMA) \\
\hline PAPI_TOT_IIS & Instructions issued (PM_INST_DISP) \\
\hline PAPI_TOT_INS & Instructions completed (PM_INST_CMPL) \\
\hline PAPI_INT_INS & $\begin{array}{l}\text { Integer instructions } \\
\text { (PM_FXU0_PROD_RESULT,PM_FXU1_PROD_RESULT,PM_FXU2_PROD_R } \\
\text { ESULT) }\end{array}$ \\
\hline PAPI_FP_INS & Floating point instructions (PM_FPU0_CMPL,PM_FPU1_CMPL) \\
\hline PAPI_LD_INS & Load instructions (PM_LD_CMPL) \\
\hline PAPI_SR_INS & Store instructions (PM_ST_CMPL) \\
\hline PAPI_BR_INS & Branch instructions (PM_BR_CMPL) \\
\hline PAPI_FLOPS & $\begin{array}{l}\text { Floating point instructions per second } \\
\text { (PM_CYC,PM_FPU0_CMPL,PM_FPU1_CMPL) }\end{array}$ \\
\hline PAPI_TOT_CYC & Total cycles (PM_CYC) \\
\hline PAPI_IPS & Instructions per second (PM_CYC,PM_INST_CMPL) \\
\hline PAPI_LST_INS & Load/store instructions completed (PM_LD_CMPL,PM_ST_CMPL) \\
\hline PAPI_SYC_INS & Synchronization instructions completed (PM_SYNC) \\
\hline PAPI_FDV_INS & Floating point divide instructions (PM_FPU_FDIV) \\
\hline PAPI_FSQ_INS & Floating point square root instructions (PM_FPU_FSQRT) \\
\hline
\end{tabular}

\section{References}

[1] J.M. Anderson, L.M. Berc, J. Dean, S. Ghemawat, M.R. Henzinger, S.-T.A. Leung, R.L. Sites, M.T. Vandevoorde, C.A. Waldspurger, and W.E. Weihl, "Continuous profiling: where have all the cycles gone?," ACM Trans. Computer Systems, 15(4):357-90, 1997.

[2] P. Bose and T.M. Conte, "Performance analysis and its impact on design," Computer, 31(5):41-9, 1998.

[3] S. Browne, J. Dongarra, N. Garner, K. London, and P. Mucci, "A Scalable CrossPlatform Infrastructure for Application Performance Tuning Using Hardware Counters," Proc. SC2000: High Performance Networking and Computing Conf. (electronic publication), 2000.

[4] J. Dean, J.E. Hicks, C.A. Waldspurger, W.E. Weihl, and G. Chrysos, "ProfileMe: hardware support for instruction-level profiling on out-of-order processors," Proc. Thirtieth Annual IEEE/ACM International Symposium on Microarchitecture (MICRO), 1997, pp. 292-302.

[5] A. Hoisie, O. Lubeck, H. Wasserman, F. Petrini, and H. Alme, "A General Predictive Performance Model for Wavefront Algorithms on Clusters of SMPs," Proc. ICPP 2000, 2000.

[6] Intel, "Intel IA-64 Architecture Software Developer's Manual, Volume 4: Itanium Processor Programmer's Guide," Intel 2000.

[7] R.A. Johnson and D.W. Wichern, Applied Multivariate Statistical Analysis, 4 ed. Englewood Cliffs, New Jersey, USA: Prentice-Hall, 1998.

[8] K.R. Koch, R.S. Baker, and R.E. Alcouffe, "Solution of the First-Order Form of the 3-D Discrete Ordinates Equation on a Massively Parallel Processor," Trans. Amer. Nuc. Soc., 65(198), 1992.

[9] K. London, J. Dongarra, S. Moore, P. Mucci, K. Seymour, and T. Spencer, "Enduser Tools for Application Performance Analysis Using Hardware Counters," Proc. International Conference on Parallel and Distributed Computing Systems, 2001. 
[10] J.M. May, "MPX: Software for Multiplexing Hardware Performance Counters in Multithreaded Programs," Proc. International Parallel and Distributed Processing Symposium (IPDPS) (electronic publication), 2001.

[11] A.A. Mirin, R.H. Cohen, B.C. Curtis, W.P. Dannevik, A.M. Dimits, M.A. Duchaineau, D.E. Eliason, D.R. Schikore, S.E. Anderson, D.H. Porter, P.R. Woodward, L.J. Shieh, and S.W. White, "Very High Resolution Simulation of Compressible Turbulence on the IBM-SP System," Proc. SC99: High Performance Networking and Computing Conf. (electronic publication), 1999.

[12] D.A. Reed, O.Y. Nickolayev, and P.C. Roth, "Real-Time Statistical Clustering and for Event Trace Reduction," J. Supercomputing Applications and HighPerformance Computing, 11(2):144-59, 1997.

[13] J.S. Vetter and F. Mueller, "Communication Characteristics of Large-Scale Scientific Applications for Contemporary Cluster Architectures," Proc. International Parallel and Distributed Processing Symposium (IPDPS), 2002.

[14] J.S. Vetter and D. Reed, "Managing Performance Analysis with Dynamic Statistical Projection Pursuit," Proc. SC99: High Performance Networking and Computing Conf. (electronic publication), 1999.

[15] M. Zagha, B. Larson, S. Turner, and M. Itzkowitz, "Performance Analysis Using the MIPS R10000 Performance Counters," Proc. Supercomputing (electronic publication), 1996. 